\title{
Adaptación y evaluación de las propiedades psicométricas de la BAS-3 para población adolescente de Tucumán (Argentina) ${ }^{*}$
}

\section{Adaptation and Evaluation of the Psychometric Properties of the BAS-3 Socialization Battery to Adolescents Sample from Tucumán (Argentina)}

\author{
Ana Betina Lacunza** \\ Silvina Valeria Caballero \\ Evangelina Norma Contini \\ Universidad Nacional de Tucumán, \\ Argentina \\ Recibido: 4 de febrero de 2012 \\ Revisado: 22 de abril de 2012 \\ Aceptado: 1 de julio de 2012
}

\section{Resumen}

Se describió la adaptación y evaluación de las propiedades psicométricas de la Batería de Socialización para Adolescentes (BAS-3) en adolescentes de Tucumán (Argentina). Se aplicó la batería a 381 adolescentes escolarizados, entre 10 y 15 años, con el previo consentimiento informado a sus padres. Se encontró una solución de dos factores que explicaban el $53,13 \%$ de la varianza. Las asociaciones estadísticas observadas dan cuenta de la presencia de factores primarios, secundarios y terciarios de la socialización. El estudio aporta datos respecto a las propiedades psicométricas del instrumento en población local, indicando su sensibilidad para la evaluación de la autopercepción de los comportamientos sociales.

Palabras clave: socialización, autopercepción, batería de socialización, adolescentes, propiedades psicométricas.

* $\quad$ Artículo de investigación. Proyecto de investigación K26-403, del Consejo de Investigaciones de la Universidad Nacional de Tucumán.

** Correspondencia: Ana Betina Lacunza: Centro Universitario Concepción (CUC), Universidad del Norte Santo Tomás de Aquino. Investigadora asistente del Consejo Nacional de Investigaciones Científicas y Técnicas (Conicet). Integrante del Proyecto de Investigación K26-403, del Consejo de Investigaciones Universidad Nacional de Tucumán. Correos electrónicos: betinalacunza@conicet.gov.ar y anabetinalacunza@ciudad.com.ar. Silvina Valeria Caballero: psicóloga y jefe de Trabajos Prácticos en la Cátedra de Evaluación Psicológica, Centro Universitario Concepción (CUC), de la Universidad del Norte Santo Tomás de Aquino. Auxiliar docente graduada de la Cátedra de Psicología Clínica, Facultad de Psicología de la Universidad Nacional de Tucumán. Integrante del proyecto de investigación K26-403, del Consejo de Investigaciones de la Universidad Nacional de Tucumán. Correo electrónico: caballero.silvinavaleria@gmail.com; Evangelina Norma Contini de González: doctora en Psicología, profesora titular de la Cátedra Teoría y Técnicas de Exploración Psicológica en Niños, de la Facultad de Psicología de la Universidad Nacional de Tucumán. Directora del proyecto de investigación K26-403, del Consejo de Investigaciones de la Universidad Nacional de Tucumán. Correo electrónico: nocon@arnet.com.ar 


\section{Abstract}

This article describes the adaptation and evaluation of psychometric properties of the BAS-3 Adolescent Socialization Battery in adolescents from Tucumán (Argentina). A total of 381 adolescents, between the ages of 10 and 15 that attend state and public schools, were evaluated with the BAS-3. The participants were administered the test with their parents' consent. A two-factor solution explaining the $53.13 \%$ of the variance was found. The statistical relations observed account for the presence of primary, secondary and third factors of socialization. The study provides data of Argentinean adolescents in relation to the psychometric properties of the test indicating its sensibility to the evaluation of social behavior self-perception.

Keywords: socialization, self-perception, socialization battery, adolescents, psychometric properties.

\section{Introducción}

Según Kirchner (1997), el interés por evaluar factores de tipo social es relativamente reciente, porque en gran parte del siglo XX, el énfasis estuvo puesto en el diagnóstico de las capacidades cognitivas. Esta autora plantea como punto de partida la década del treinta, con los estudios sobre el comportamiento asertivo en niños (Murphy, Murphy y Newcomb, 1937; Williams, 1935), con un resurgimiento en los años sesenta y setenta, focalizándose los estudios desde una perspectiva cognitivo-conductual (Bandura, 1976; Pelechano Barberá, 1979). Tanto los estudios iniciales como los contemporáneos sobre el comportamiento social han destacado la influencia que tienen las variables psicosociales en la conformación de la personalidad y en el ajuste del sujeto al medio. Es así como proliferaron instrumentos centrados, principalmente, en evaluar los aspectos comportamentales claves en la interacción social, aunque con diferencias conceptuales y metodológicas.

Estas diferencias ya habían sido planteadas por Curran (1979), al considerar que las habilidades sociales son un megaconstructo en el que se tiende a relacionar una variedad de comportamientos superficialmente verosímiles. Por ello, su evaluación supone la revisión de teorías psicométricas y estrategias metodológicas que permitan evaluar no solo los aspectos comportamentales, sino además los factores cognitivos y los estados emocionales involucrados en las capacidades sociales. En este sentido, Michelson, Sugai, Wood y Kazdin
(1987) sugieren que las principales técnicas sobre habilidades sociales son las pruebas de observación de la conducta, heteroinformes y autoinformes; mientras que Monjas Casares (2000) agrega a este listado la utilización de entrevistas.

Esta variedad de pruebas pueden brindar un perfil más exhaustivo sobre las capacidades sociales de un sujeto, si se las utiliza dentro de una perspectiva multimétodo y multiinformante. La propuesta multimétodo y multiinformante es una característica propia de la evaluación infanto-juvenil (Forns I Santacana, 1993) y ha sido empleada en múltiples estudios psicológicos (Figueroa Masip, 2006; Giraldo Alarcón, 2010; Lacunza, 2007; Maganto y Cruz, 2001; Reynolds y Komphons, 1992; Schelton y Frick I Wootton, 1996; Valero, 1997). El concepto de multimétodo tiene su origen en los trabajos de Campbell y Fiske (1959) sobre las matrices multimétodo-multirrasgo; posteriormente, Denzin (1978) propuso que el multimétodo es una forma de triangulación, puesto que combina métodos cuantitativos y cualitativos. Hernández, Plaza, Pozo y Alonso (2004) analizan la postura de distintos autores y sostienen que la aproximación multimétodo es una opción apropiada en la investigación social, puesto que plantea una especie de conversación entre distintos paradigmas y aproximaciones metodológicas dentro de un mismo estudio.

Desde una perspectiva cuantitativa, los autoinformes suelen ser uno de los instrumentos más utilizados para operacionalizar constructos sobre 
las interrelaciones sociales. Si bien son prácticos, sencillos y económicos para contar con una estimación de la autopercepción sobre los recursos sociales, están sometidos a la distorsión y subjetividad inherente a toda autoevaluación (Kirchner, 1997). El hecho de que el propio sujeto evaluado sea la fuente de información ha merecido una serie de críticas y advertencias (Pérez-Pareja, 2007). Esto ha llevado a que se proponga la contrastación entre las medidas de autoinforme y los heteroinformes, dentro de la perspectiva ya definida como multimétodo y multiinformante.

Son múltiples los autoinformes sobre habilidades sociales desarrollados para población infanto-juvenil. Entre los diseñados o adaptados al castellano se mencionan el Cuestionario de Habilidades de Interacción Social (CHIS) (Monjas, 2000); la Matson Evaluation of Social Skills with Youngsters (MESSY) (Matson, Rotatori y Helsel, 1983), versión de autoinforme adaptada recientemente por Ipiña, Molina y Reyna (2011) para población de Córdoba (Argentina); la Escala de Habilidades Sociales de Gismero (Gismero, 2000); la Escala de Autoeficacia Social para Universitarios (EAS-U) (Olaz, 2008), adaptada para población adolescente (Sheinsohn, Freiberg y Luchetti, 2011); y la Batería de Socialización (BAS-3) (Silva Moreno y Martorell Pallás, 2001), adaptada por Gargordobil para adolescentes tempranos (Garaigordobil, 2003). Aunque algunos de estos instrumentos ya fueron adaptados para el contexto local (tal como es el caso de la escala MESSY y la EAS-U), subsiste una vacancia respecto al análisis de las propiedades psicométricas y la adecuación de los instrumentos para población adolescente del Norte de Argentina.

\section{La Batería de Socialización (BAS)}

La BAS surge en la década del ochenta con antecedentes conceptuales y metodológicos bien definidos. Desde lo conceptual, sus autores realizaron revisiones de publicaciones y aportes teóricos, como la teoría bifactorial de Kohn (1977) (Martorell, 1997). Este autor, basándose en estudios sistemáticos previos, planteó dos factores bien identificados del constructo socialización: re- traimiento y agresividad - conducta antisocial, los que desde un análisis psicopatológico representan dos núcleos problemáticos: problemas de conducta (agresividad antisocial) y problemas de personalidad (retraimiento o inhibición). Actualmente, estas dimensiones, desde la psicopatología infanto-juvenil, se denominan "externalización e internalización” (Achenbach, 1992, 1995). Este último factor propuesto por Achenbach y Edelbrock (1978) posee gran similitud con los postulados por Kohn (1977) respecto a que los problemas de personalidad (inseguridad, retraimiento, timidez) se ubicarían en el polo de internalización, mientras que los problemas de conducta (desobediencia, conducta disruptiva, agresión, hiperactividad) lo harían en la dimensión en el polo de externalidad.

Desde lo metodológico pueden mencionarse los estudios de los autores Silva Moreno y Martorell Pallás con la Escala de Adaptación y Hábitos Sociales (AHS-1) y la Escala de Trastornos de Conducta (ETC-1) (Silva y López de Silva, 1979), como también las Escalas de Socialización Escolar de Aspectos Facilitadores (ESE-1) e Inhibidores (ESE-2) (Silva, Martorell y Pelechano, 1981). La ESE-1 incluye cuatro factores positivos de la socialización: colaboración, respeto y responsabilidad, popularidad y liderazgo como actitud diferencial positiva entre actividades escolares y extraescolares. Por su parte, la ESE-2 evalúa hiperactividad $y$ agresividad e inseguridad y retraimiento.

La BAS estudia la conducta social de niños y adolescentes a partir de la percepción de profesores (BAS-1), padres (BAS-2) y del sujeto mismo (BAS3). Específicamente, la BAS-3 permite lograr un perfil de la conducta social en adolescentes de 11 a 19 años, en cinco escalas de socialización:

1. Consideración con los demás (Co): detecta sensibilidad social o preocupación por los demás, en particular por aquellos que tienen problemas y son rechazados y postergados.

2. Autocontrol en las relaciones sociales (Ac): evalúa, en su polo positivo, acatamiento de reglas y normas sociales que facilitan la convivencia en el mutuo respeto, y en el polo 
negativo, conductas agresivas, impositivas y de terquedad e indisciplina.

3. Retraimiento social (Re): identifica alejamiento tanto pasivo como activo de los demás, hasta llegar, en el extremo, a un claro aislamiento.

4. Ansiedad social y timidez (At): mide distintas manifestaciones de ansiedad (por ejemplo, miedo, nerviosismo), unidas a reacciones de timidez (retraimiento, vergüenza) en las relaciones sociales.

5. Liderazgo (Li): explora ascendencia, popularidad, iniciativa, confianza en sí mismo y espíritu de servicio.

A estas escalas se suma una sexta: sinceridad (S). Los autores afirman que esta escala no debe ser interpretada en el sentido sinceridad vs. simulación, sino como indicadora de inconformismo social, desconocimiento y desacatamiento de normas sociales.

El concepto de socialización que explora la batería BAS comprende desde una serie de habilidades básicas hasta patrones conductuales más amplios de relación interpersonal y grupal (Kirchner, 1997). Esta posición es concordante con las perspectivas sobre socialización planteadas desde la década del setenta, en las que se incluían aspectos relacionados con el propio yo y el de los demás, es decir, la connotación de lo interpersonal (Martorell, González, Aloy y Ferris, 1995).

Silva Moreno y Martorell Pallás (1991) realizaron una taxonomía de los patrones de comportamiento social a partir de una factorialización de la BAS-3, extrayendo las dimensiones básicas de la socialización. Afirmaron la existencia de una estructura jerárquica de la socialización que respondía básicamente a dos dimensiones: conducta prosocial vs. conducta antisocial, y sociabilidad vs. baja sociabilidad. La primera dimensión (conducta prosocial vs. conducta antisocial) contenía en su polo positivo a las escalas consideración con los demás y autocontrol en las relaciones sociales, mientras que en el polo negativo (conducta antisocial) se ubicaba agresividad. Por su parte, en la dimensión sociabilidad se encontraba liderazgo, en tanto en el extremo negativo de esta dimensión (baja sociabilidad) se situaba ansiedad social/timidez y retraimiento (figura 1).

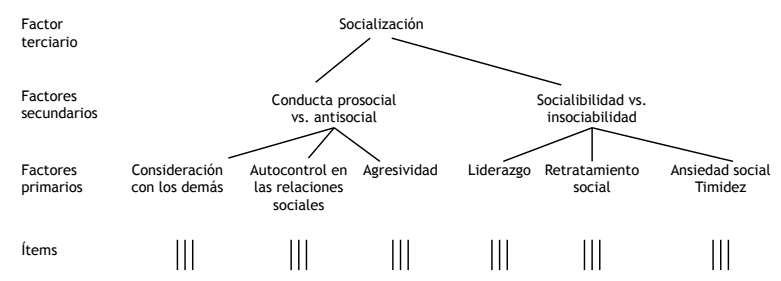

Figura 1. Estructura de las dimensiones de la socialización.

Fuente: Silva Moreno y Martorell Pallás (1991).

González Portal (1992) resume este modelo indicando que se trata de una estructura claramente jerárquica. En la base se encuentran los factores primarios, compuestos por las diferentes escalas de la BAS-3 (consideración con los demás, autocontrol en las relaciones sociales, liderazgo, retraimiento social y ansiedad social/timidez); en el polo negativo de la escala se encuentra autocontrol en las relaciones sociales, es decir, agresividad; la parte superior de estas escalas se agrupan en dos factores secundarios: conducta prosocial vs. conducta antisocial, y sociabilidad vs. insociabilidad, ubicándose en la cúspide el factor terciario: la socialización propiamente dicha.

A nivel operacional, los factores primarios de la socialización están contemplados en los distintos ítems de la BAS-3; es decir, los 65 ítems de la batería (sin contar los 10 ítems incluidos en la escala de sinceridad) son un repertorio de habilidades sociales que se presentan al adolescente al momento de su evaluación. Dichas habilidades sociales ponen su acento en el componente conductual y/o sociocognitivo. Desde la concepción conductual, las habilidades sociales son respuestas adquiridas por el aprendizaje, ligadas a contextos específicos que sirven para resolver problemas interpersonales inmediatos y específicos de la situación (Caballo, 2005; Michelson et ál., 1987; Monjas, 2000). 
Por su parte, el enfoque cognitivo trata de operativizar las dimensiones cognitivas del comportamiento interpersonal eficaz, por lo que aunque las habilidades sociales se aprenden en contextos determinados, estas pueden aplicarse a diferentes situaciones (González y Pelechano, 1996). Si bien los ítems de la BAS-3 enuncian comportamientos sociales específicos (por ejemplo, el ítem 31: juego más con los otros que solo; ítem 34: soy violento y golpeo a los demás; ítem 40: grito y chillo con facilidad; ítem 65: protesto cuando me mandan a hacer algo; ítem 72: me aparto cuando hay muchas personas juntas, entre otros) que podrían atribuirse solo a la mirada conductual, su desempeño requiere cierto compromiso cognitivo de generación de alternativas, planificación, pensamiento secuencial, entre otros, que permitan la resolución de la situación interpersonal.

A su vez, las dimensiones primarias y secundarias de la socialización propuestas desde el modelo jerárquico de Martorell et ál. (1995) pueden entenderse desde las variables interpersonales de la personalidad. Silva Moreno y Martorell Pallás (1991) analizaron las propiedades psicométricas de la BAS-3 (con modificaciones en sus múltiples alternativas de respuesta) en una muestra de 1269 niños mayores y adolescentes de España. Las dimensiones secundarias de la socialización conducta prosocial vs. conducta antisocial, como también la de sociabilidad vs. baja sociabilidad, se analizaron desde un sentido divergente más que convergente. Con base en los gráficos de los análisis factoriales realizados, se representaron dos ejes y cuatro polos que conformaban un modelo de aspa (Silva Moreno, Moro y Ortet, 1994).

El eje conducta prosocial vs. conducta antisocial estaba compuesto, en su polo positivo, por las escalas consideración con los demás y respeto/ autocontrol en las relaciones sociales, mientras que en el polo negativo se ubicaba agresividad - conducta antisocial. El eje sociabilidad - baja sociabilidad (insociabilidad) incluía en su polo positivo a las escalas liderazgo, ascendencia social y jovialidad; en tanto que en el polo negativo se incluía retraimiento social y ansiedad social/timidez. Si se tienen en cuenta las escalas descritas en el manual de la BAS-3, hay que advertir que la escala autocontrol en las relaciones sociales se desdobló en su polo negativo de agresividad - conducta antisocial y en su polo positivo de respeto - autocontrol en las relaciones sociales, siguiendo de este modo las tendencias descritas en la BAS 1 y 2 (Cordero, 1997).

Con ello se dio lugar a seis escalas, las que ya habían sido descritas en el modelo inicial de socialización. Puesto que los ítems que representan la escala jovialidad no están incluidos en la BAS3 (pero sí en BAS-1 y 2), los autores supusieron que ubicarían esta escala en el polo positivo de la sociabilidad, de modo opuesto a retraimiento social, ya que esta mide la extraversión en sus connotaciones concretas de sociabilidad y buen ánimo (Silva Moreno y Martorell Pallás, 1983). Los polos descritos contienen un sentido positivo y otro negativo de la orientación interpersonal. La sociabilidad representa la aproximación a los demás, mientras que la baja sociabilidad, el distanciamiento. Por su parte, la conducta prosocial da cuenta de un ser para los demás, mientras que la conducta antisocial muestra un ser contra los demás (Silva Moreno, Martínez Arias y Ortet, 1997). Estas vertientes de la orientación interpersonal conectan las dimensiones de la socialización con ciertas variables de personalidad, particularmente aquellas del área interpersonal.

Distintos estudios han utilizado la BAS-3 en población adolescente. Tal es el caso de Garaigordobil (2003), que realizó una adaptación de la BAS-3 con una muestra de 139 niños españoles, de 10 a 12 años. El autor propuso una reducción de la escala a 65 ítems (eliminando la escala sinceridad) y una modificación de las opciones de respuesta dicotómicas a opciones (nada, algo, bastante, mucho), con puntajes de 0 a 3. El análisis de fiabilidad de esta versión mostró que la consistencia interna fue satisfactoria (alfa de Cronbach entre 0,73 y 0,82 ); la estabilidad temporal (test-retest en intervalo de cuatro meses) fue satisfactoria para autocontrol $(r=0,66)$ y liderazgo $(r=0,61)$, aunque fue baja en consideración $(r=0,42)$ y retraimiento $(r=0,43)$. Respecto a la validez, estudios en menores no-delincuentes y delincuentes internos observaron que los menores delincuentes tenían puntuaciones más bajas en conside- 
ración con los demás y autocontrol, y más altas en retraimiento. La escala consideración con los demás obtuvo correlaciones positivas con empatía $(r=0,42)$ y negativas con psicoticismo $(r=$ $-0,37)$; la escala autocontrol mostró correlaciones negativas con psicoticismo $(r=-0,44)$, conducta antisocial $(r=-0,40)$ e impulsividad $(r=-0,43)$; la escala retraimiento tuvo correlaciones negativas con extraversión $(r=-0,36)$; la escala ansiedad correlacionó con neuroticismo $(r=0,40)$, y la de liderazgo, con extraversión $(r=0,27)$ (Silva Moreno y Martorell Pallás, 2001).

Por su parte, Lacunza (2010) analizó las propiedades psicométricas de la BAS-3 en una muestra de 165 niños de $4^{\circ}$ y $5^{\circ}$ grado de nivel primario (edad promedio de 10 años) de la ciudad de San Miguel de Tucumán. Los estudios de fiabilidad indicaron que la consistencia interna de las escalas fue satisfactoria (coeficiente alfa de Cronbach entre 0,523 a 0,734 para las distintas escalas), en tanto los análisis factoriales exploratorios explicaban la interrelación de ítems en torno a las dimensiones facilitadoras e inhibidoras. En la misma línea, Smulovitz (2011) analizó las propiedades psicométricas de la prueba en niños de 9 y 10 años de una ciudad del sur de la provincia de Tucumán. Halló que un análisis factorial exploratorio (rotación Varimax, índice de adecuación muestral de Kaiser-Meyer-Olkin $=0,739$, prueba de esfericidad de Bartlett $=$ X2 = 6191, 279 [gl = 2775], $\mathrm{p}<0,000$ ) discriminaba cinco factores con el $27 \%$ de la varianza (se eliminaba la escala retraimiento). Los factores descritos mostraban niveles de fiabilidad aceptables, oscilando entre 0,564 y 0,716.

Los objetivos de este trabajo fueron: a) analizar las propiedades psicométricas de la Batería de Socialización BAS-3 en adolescentes de San Miguel de Tucumán (Argentina); y b) identificar las dimensiones de la socialización propuestas desde el modelo jerárquico.

\section{Método}

Se trató de un estudio instrumental (CarreteroDios y Pérez, 2007; Montero y León, 2005) a través del cual se estudiaron las propiedades psico- métricas de la Batería de Socialización BAS-3 y las dimensiones de la socialización propuestas en el modelo jerárquico.

\section{Participantes}

En la muestra intencional participaron 381 adolescentes, de 10 a 15 años escolarizados en instituciones públicas y privadas de la ciudad de San Miguel de Tucumán (Argentina). Se incluyeron aquellos adolescentes escolarizados en el último año del nivel primario y primer año del ciclo secundario, que no presentaran antecedentes de problemas de socialización según informante clave (docente y profesional del gabinete psicopedagógico). La edad promedio fue de 12 años $(X=11,56, D E=0,896)$. El $51 \%$ de la muestra eran mujeres y el $66 \%$ asistía a instituciones educativas públicas.

\section{Instrumentos}

La Batería de Socialización BAS-3 (Silva Moreno y Martorell Pallás, 2001) está integrada por 75 ítems, aplicable a adolescentes de 11 a 19 años de edad, de autoadministración individual con dos opciones de respuesta (sí, no). Permite obtener un perfil del comportamiento social a partir de sus cinco escalas de socialización: consideración con los demás (Co), autocontrol en las relaciones sociales (Ac), retraimiento social (Re), ansiedad social/timidez (At) y liderazgo (Li). A estas se le suma una escala de sinceridad (S), que permite analizar la consistencia interna de las respuestas a las escalas. Por cada escala se obtiene una puntuación bruta que se transforma en percentiles, según un baremo propuesto por los autores de la prueba, a partir de una muestra de 1770 adolescentes españoles de nivel socioeconómico medio y medio-bajo. Las propiedades psicométricas de la versión original se basaron en una muestra de 806 adolescentes. Se realizaron dos estudios de fiabilidad: consistencia interna y test-retest; los valores de consistencia interna oscilaron entre 0,73 y 0,82 , siendo menor en la escala $S(0,60)$. La estabilidad temporal mostró resultados variables: índices satisfactorios para Ac, At, Li y S, pero menor en $\mathrm{Co}(0,42)$ y $\operatorname{Re}(0,43)$. 


\section{Procedimiento}

Se efectuó una aplicación piloto de la prueba en 20 adolescentes pertenecientes a diversos niveles socioeconómicos de San Miguel de Tucumán, a fin de evaluar la claridad de los ítems y comprensión de la consigna, así como de las expresiones lingüísticas utilizadas. Tal como lo plantea Cohen y Swerdlik (2001), las condiciones de aplicación fueron lo más similares posible a las condiciones bajo las cuales se aplicó luego la versión definitiva de la batería. Los adolescentes no solo respondieron al instrumento, sino que además indicaron aquellos ítems que aparecían como confusos o poco comprensibles. Fueron recurrentes las dificultades para la compresión de algunos términos. A partir de estos resultados se realizaron adecuaciones lingüísticas a los ítems $13,26,28$, $48,51,53,67$ y 75 de la versión original, tratando de mantener la equivalencia de los conceptos respecto a la cultura donde fue desarrollado el instrumento y la cultura destinataria.

Posteriormente, el protocolo fue validado por el sistema de jueces, a fin de identificar la concordancia entre el ítem original y la modificación lingüística. A cinco profesionales con experiencia en psicología evolutiva y evaluación psicológica se les solicitó una valoración a ciegas de los ítems. Teniendo en cuenta las apreciaciones de los especialistas, se decidió la inclusión de los ítems que integrarían la versión local. Esta nueva versión fue aplicada a una muestra piloto de 30 adolescentes residentes en San Miguel de Tucumán. Este estudio demostró que los ítems eran bien comprendidos y que la consigna no generaba dificultades.

Utilizando esta versión modificada en lo lingüístico, se aplicó la BAS-3 a los participantes, a fin de evaluar sus propiedades psicométricas. Se analizó la confiabilidad a partir de la consistencia interna (coeficiente alfa) y la validez de constructo, a partir de la evidencia basada en la estructura interna del test (Tornimbeni, Pérez y Olaz, 2008), desde un análisis factorial exploratorio de componentes principales con rotación Varimax. Además, se analizaron los puntajes brutos de las distintas escalas de la prueba respecto a las dimensiones de socialización propuestas por los autores españoles. Se utilizó el programa SPSS 11.5.

\section{Resultados}

En un primer momento se analizó la validez de constructo de la BAS-3 en población adolescente de Tucumán. Se utilizó un análisis factorial exploratorio de componentes principales con rotación Varimax (índice de adecuación muestral de Kaiser-Meyer-Olkin $=0,674$; test de esfericidad de Bartlett $=X^{2}=6984.664[\mathrm{gl}=2775, \mathrm{p}<0,000]$ ). El análisis del gráfico de sedimentación mostró como mejor solución aquella que agrupaba a los ítems en cinco factores (tabla 1), puesto que no se incluyeron los ítems correspondientes a la escala de sinceridad. Esta solución explicaba el $26,65 \%$ de la varianza total y era congruente con la propuesta de los autores de la BAS-3. El factor 1 coincidió con la escala ansiedad social/timidez, incluyendo además los ítems 8, 52, 69 y 72 (incluidos según la versión original española en la escala Re); el factor 2 se denominó autocontrol en las relaciones sociales y contuvo a los ítems 3, 16, 59 (escala Co) y 23 (escala Li); el factor 3 refirió la escala retraimiento; el factor 4 , consideración con los demás; y el factor 5 , liderazgo. Si bien en el factor 2 se observaron ítems con una saturación menor a 0,20 , se los conservó por su aproximación a este valor, en tanto que el ítem 47 se mantuvo por la escasa cantidad de ítems que componían al factor 3 . 
Tabla 1.

Solución factorial, rotación Varimax e ítems escala BAS-3 $(n=381)$

\begin{tabular}{|c|c|c|c|c|c|}
\hline $\begin{array}{l}\text { Ítems } \\
\text { BAS-3 }\end{array}$ & $\begin{array}{l}\text { Factor } 1 \\
\text { (17 items) }\end{array}$ & $\begin{array}{l}\text { Factor } 2 \\
\text { (18 items) }\end{array}$ & $\begin{array}{c}\text { Factor } 3 \\
(9 \text { items })\end{array}$ & $\begin{array}{c}\text { Factor } 4 \\
\text { (11 items) }\end{array}$ & $\begin{array}{l}\text { Factor } 5 \\
(10 \text { items })\end{array}$ \\
\hline Ítem 1 & 0,351 & & & & \\
\hline Ítem 7 & 0,283 & & & & \\
\hline Ítem 18 & 0,449 & & & & \\
\hline Ítem 19 & 0,404 & & & & \\
\hline Ítem 28 & 0,443 & & & & \\
\hline Ítem 33 & 0,323 & & & & \\
\hline Ítem 38 & 0,566 & & & & \\
\hline Ítem 39 & 0,472 & & & & \\
\hline Ítem 43 & 0,579 & & & & \\
\hline Ítem 48 & 0,491 & & & & \\
\hline Ítem 52 & 0,288 & & & & \\
\hline Ítem 54 & 0,557 & & & & \\
\hline Ítem 55 & 0,402 & & & & \\
\hline Ítem 57 & 0,451 & & & & \\
\hline Ítem 62 & 0,510 & & & & \\
\hline Ítem 69 & 0,265 & & & & \\
\hline Ítem 72 & 0,394 & & & & \\
\hline Ítem 3 & & 0,340 & & & \\
\hline Ítem 4 & & 0,458 & & & \\
\hline Ítem 13 & & 0,197 & & & \\
\hline Ítem 16 & & 0,181 & & & \\
\hline Ítem 22 & & 0,368 & & & \\
\hline Ítem 23 & & 0,302 & & & \\
\hline Ítem 34 & & 0,380 & & & \\
\hline Ítem 36 & & 0,552 & & & \\
\hline Ítem 40 & & 0,366 & & & \\
\hline Ítem 44 & & 0,562 & & & \\
\hline Ítem 46 & & 0,425 & & & \\
\hline Ítem 50 & & 0,361 & & & \\
\hline Ítem 53 & & 0,485 & & & \\
\hline Ítem 56 & & 0,142 & & & \\
\hline Ítem 59 & & 0,380 & & & \\
\hline Ítem 64 & & 0,482 & & & \\
\hline Ítem 65 & & 0,480 & & & \\
\hline Ítem 73 & & 0,327 & & & \\
\hline Ítem 9 & & & 0,484 & & \\
\hline Ítem 25 & & & 0,516 & & \\
\hline Ítem 30 & & & 0,387 & & \\
\hline Ítem 31 & & & 0,203 & & \\
\hline Ítem 35 & & & 0,319 & & \\
\hline Ítem 37 & & & 0,411 & & \\
\hline Ítem 42 & & & 0,417 & & \\
\hline Ítem 47 & & & 0,149 & & \\
\hline Ítem 63 & & & 0,436 & & \\
\hline Ítem 5 & & & & 0,287 & \\
\hline
\end{tabular}

\begin{tabular}{|c|c|c|c|c|c|}
\hline $\begin{array}{l}\text { Ítems } \\
\text { BAS-3 }\end{array}$ & $\begin{array}{l}\text { Factor } 1 \\
\text { (17 items) }\end{array}$ & $\begin{array}{c}\text { Factor } 2 \\
(18 \text { items })\end{array}$ & $\begin{array}{c}\text { Factor } 3 \\
(9 \text { items })\end{array}$ & $\begin{array}{c}\text { Factor } 4 \\
(11 \text { items }\end{array}$ & $\begin{array}{l}\text { Factor } 5 \\
(10 \text { items })\end{array}$ \\
\hline Ítem 11 & & & & 0,442 & \\
\hline Ítem 12 & & & & 0,544 & \\
\hline Ítem 14 & & & & 0,531 & \\
\hline Ítem 17 & & & & 0,353 & \\
\hline Ítem 27 & & & & 0,526 & \\
\hline Ítem 51 & & & & 0,578 & \\
\hline Ítem 60 & & & & 0,421 & \\
\hline Ítem 61 & & & & 0,235 & \\
\hline Ítem 66 & & & & 0,400 & \\
\hline Ítem 68 & & & & 0,546 & \\
\hline Ítem 2 & & & & & 0,392 \\
\hline Ítem 6 & & & & & 0,555 \\
\hline Ítem 10 & & & & & 0,323 \\
\hline Ítem 20 & & & & & 0,482 \\
\hline Ítem 21 & & & & & 0,429 \\
\hline Ítem 26 & & & & & 0,326 \\
\hline Ítem 29 & & & & & 0,513 \\
\hline Ítem 45 & & & & & 0,411 \\
\hline Ítem 70 & & & & & 0,335 \\
\hline Ítem 71 & & & & & 0,408 \\
\hline $\begin{array}{l}\% \text { de } \\
\text { varianza } \\
\text { explicada }\end{array}$ & 8,88 & 5,56 & 4,23 & 3,78 & 2,85 \\
\hline
\end{tabular}

Fuente: autores.

Por otro lado, se analizó la confiabilidad de las distintas escalas de la prueba utilizando el coeficiente alfa de Cronbach. La consistencia interna de la escala consideración fue de 0,688 ; autocontrol, de 0,676; retraimiento, de 0,685; ansiedadtimidez, de 0,721; liderazgo, de 0,520; y sinceridad, de 0,608 . No se determinó un índice total puesto que la prueba no establece un puntaje total de la batería, sino solo por escalas.

El segundo objetivo fue identificar las dimensiones de la socialización incluidas en el modelo jerárquico. Para ello se consideraron como indicadores de la dimensión conducta prosocial los puntajes brutos de las escalas Co y la sumatoria de los ítems positivos de la escala Ac, mientras que la conducta antisocial fue operacionalizada desde la sumatoria de los ítems negativos de Ac, atribuyéndose valor positivo a aquellos ítems en los que los participantes respondían positivamente, es decir, de modo contrario a como fue la propuesta original. Por su parte, la dimensión sociabilidad estuvo formada por los puntajes en la escala $\mathrm{Li}$, en tanto que la baja sociabilidad se 
componía de la sumatoria de Re y At. A su vez, se determinó un indicador de la socialización a partir de la sumatoria de la dimensión positiva de la conducta prosocial y la de sociabilidad.

Se efectuaron sucesivos análisis factoriales exploratorios de componentes principales y rotación Varimax de las mencionadas escalas (sin incluir la escala S), observándose una solución de dos factores (con rotación Varimax) que explicaban el 53,13\% de la varianza (índice de adecuación muestral de Kaiser-Meyer-Olkin $=0,583$, test de esfericidad de Bartlett $=X^{2}=302.664$ [gl $\left.=15\right]$, $\mathrm{p}<0,000)$. Esta solución indicaba dos factores: el primero reunía las escalas Co y Ac en su polo positivo, como la escala Ag lo hacía en su polo negativo. Este factor fue coincidente con el denominado conducta prosocial vs. conducta antisocial. El segundo factor incluía a At y Re en su polo positivo y Li en su polo negativo, lo que si bien es congruente a la bipolaridad sociabilidad vs. baja sociabilidad, ubicaba a liderazgo en un polo negativo (tabla 2).

Tabla 2.

Solución factorial, rotación Varimax, dimensiones de la socialización $(n=381)$

\begin{tabular}{|c|c|c|}
\hline Escalas BAS-3 & $\begin{array}{c}\text { Factor } \\
1\end{array}$ & $\begin{array}{c}\text { Factor } \\
2\end{array}$ \\
\hline Consideración con los demás & 0,592 & $-0,351$ \\
\hline $\begin{array}{l}\text { Autocontrol en las relaciones } \\
\text { sociales }\end{array}$ & 0,849 & 0,34 \\
\hline Liderazgo & 0,189 & $-0,306$ \\
\hline Retraimiento & $-0,153$ & 0,805 \\
\hline Ansiedad social - timidez & $-0,003$ & 0,818 \\
\hline Agresividad & $-0,704$ & 0,168 \\
\hline$\%$ varianza explicada & $34,59 \%$ & $18,53 \%$ \\
\hline
\end{tabular}

Fuente: autores.

Se calcularon las intecorrelaciones entre las distintas escalas de la BAS-3, incluidas la de sinceridad, a fin de comprobar su correspondencia a las dimensiones bipolares de la socialización. Se encontraron asociaciones estadísticas significati- vas entre las escalas $\mathrm{Co}, \mathrm{Ac}$ y $\mathrm{Li}$, descritas como facilitadoras de la socialización (Silva Moreno y Martorell Pallás, 2001). A su vez, Co, escala perteneciente al primer factor secundario (conducta prosocial), se asoció negativamente con Ag $(r=$ $-0,231, p<0,00)$ y en mayor medida con $\operatorname{Re}(r$ $=-0,348, p<0,00$ ), escala perteneciente al segundo factor secundario de la socialización (baja sociabilidad). Por su parte, se observaron asociaciones estadísticas moderadas entre Re y At $(r=$ $0,433, p<0,00$ ), pertenecientes al polo negativo de la dimensión sociabilidad - baja sociabilidad, y de estas con respecto a Ag (Re-Ag: $r=0,192$, $p<0,00$; At-Ag: $r=0,247, p<0,00)$. La correlación negativa entre Ac y Ag $(r=-0,410, p<0,00)$ sostenía la bipolaridad de la dimensión conducta prosocial vs. conducta antisocial (tabla 3).

Tabla 3.

Intecorrelaciones de las escalas BAS-3 $(n=381)$

\begin{tabular}{ccccccc}
\hline $\begin{array}{c}\text { Escalas } \\
\text { BAS-3 }\end{array}$ & Ac & Li & Re & At & Ag & S \\
\hline Co & $0,317^{* *}$ & $0,248^{* *}$ & $-0,348^{* *}$ & $-0,107^{*}$ & $-0,231^{* *}$ & $-0,166^{* *}$ \\
AC & 1 & 0,075 & $-0,148^{* *}$ & $-0,081$ & $-0,410^{* *}$ & $-0,340^{* *}$ \\
Li & & 1 & $-0,076$ & $-0,123^{*}$ & $-0,031$ & $-0,198^{* *}$ \\
$\operatorname{Re}$ & & & 1 & $0,433^{* *}$ & $0,192^{* *}$ & $-0,106^{*}$ \\
At & & & & 1 & $0,247^{* *}$ & 0,010 \\
Ag & & & & & 1 & $0,379^{* *}$ \\
S & & & & & & 1 \\
\hline
\end{tabular}

Nota: ${ }^{*} \mathrm{p}<0,05$

${ }^{* *} p<0,01$

Fuente: autores.

Un análisis de los puntajes promedio mostró puntuaciones superiores en el polo negativo baja sociabilidad $(X=8,04, D E=4,5)$, respecto al positivo de la sociabilidad $(X=7,62, D E=2,34)$ (tabla 4). Cabe destacar que el puntaje promedio en el factor terciario socialización fue de 23 puntos $(X=$ 23,39, $D E=434)$. 
A su vez, se analizaron las asociaciones estadísticas entre los factores encontrados, es decir, las dimensiones de la socialización, las escalas de la BAS-3 y el indicador de socialización obtenido desde el modelo jerárquico. Se halló que Co y Li tenían una asociación estadística altamente significativa con la socialización, siendo moderada esta asociación respecto a Ac. Por su parte, $\mathrm{Ag}$, Re y At presentaban una relación estadística negativa con la socialización. Respecto a la dimensión socialización, la conducta prosocial vs. conducta antisocial correlacionaba positivamente con Co, Ac y en menor medida con Li (perteneciente al polo negativo de esta dimensión), mientras se asociaba negativamente con $\mathrm{Ag}$ y $\operatorname{Re}$ (incluidas en la segunda dimensión). La polaridad sociabilidad vs. baja sociabilidad se relacionaba estadísticamente con Li y en menor medida con Co (escala de la primera dimensión) y en su polo negativo con Re y At. También se observó una asociación estadística con Ag (indicador de la dimensión conducta prosocial vs. conducta antisocial). Se incluyó la escala $S$, encontrándose una relación estadística positiva con la conducta antisocial y negativa con la conducta prosocial y la sociabilidad (tabla 4).

Tabla 4.

Correlaciones entre factores primarios, secundarios y terciarios de la socialización.

Modelo jerárquico $(n=381)$

\begin{tabular}{|c|c|c|c|c|c|}
\hline \multirow{2}{*}{$\begin{array}{c}\text { Factores primarios } \\
\text { de la escalas } \\
\text { BAS-3 }\end{array}$} & \multicolumn{4}{|c|}{$\begin{array}{l}\text { Factores secundarios } \\
\text { Dimensiones de socialización }\end{array}$} & \multirow{2}{*}{ Socialización } \\
\hline & $\begin{array}{l}\text { Conducta } \\
\text { prosocial }\end{array}$ & $\begin{array}{l}\text { Conducta } \\
\text { antisocial }\end{array}$ & Sociabilidad & $\begin{array}{c}\text { Baja } \\
\text { sociabilidad }\end{array}$ & \\
\hline $\begin{array}{l}\text { Consideración con los } \\
\text { demás }\end{array}$ & $0,875^{* *}$ & $-0,231^{\text {** }}$ & $0,284^{* *}$ & $-0,254^{* *}$ & $0,784^{* *}$ \\
\hline Autocontrol & $0,736^{* *}$ & $-0,410^{* *}$ & 0,075 & $-0,132^{* *}$ & $0,572^{* *}$ \\
\hline Liderazgo & $0,241^{* *}$ & $-0,031$ & 1 & $-0,119^{*}$ & $0,714^{* *}$ \\
\hline Retraimiento & $-0,324^{* *}$ & $0,192^{* *}$ & $-0,076$ & $0,821^{* *}$ & $-0,274^{* *}$ \\
\hline $\begin{array}{l}\text { Ansiedad social - } \\
\text { timidez }\end{array}$ & $-0,117^{*}$ & $0,247^{* *}$ & $-0,123^{*}$ & $0,870^{* *}$ & $-0,151^{* *}$ \\
\hline Agresividad & $-0,374^{* *}$ & 1 & $-0,031$ & $0,262^{* *}$ & $-0,286^{* *}$ \\
\hline Sinceridad & $-0,292^{* *}$ & $0,379^{* *}$ & $-0,198^{* *}$ & $-0,051$ & $-0,317^{* *}$ \\
\hline
\end{tabular}

Nota: ${ }^{*} p<0,05$

${ }^{* *} p<0,01$

Fuente: autores.

\section{Discusión}

El primer objetivo de este estudio fue describir las propiedades psicométricas de la BAS-3 en población adolescente de Tucumán. Se analizó la validez de constructo a partir de análisis factoriales exploratorios de las escalas de la prueba y de las dimensiones de la socialización, encontrándose, en un primer momento, una solución factorial que agrupaba los 65 ítems (exceptuando los correspondientes a la escala S) en cinco factores. Estos factores reproducían las escalas de socialización 
At, Ac, Re, Co y Li propuestas en la BAS-3. Sin embargo, se encontraron diferencias respecto a la cantidad de ítems, es decir, el conjunto de habilidades sociales incluidas en cada factor. El factor 1 (ansiedad social - timidez) contuvo a los ítems de la BAS-3 y a otros cuatro ítems (ítem 8: evito a los demás; ítem 52: intento estar en lugares apartados, poco visibles o concurridos; ítem 69: permanezco sentado sin enterarme de nada, durante mucho tiempo; ítem 72: me aparto cuando hay muchas personas juntas), por lo que quedaba conformado por un total de 17 ítems, a diferencia de los 12 de la versión española.

Según la percepción de los adolescentes tucumanos, evitar a otros sujetos $u$ otras situaciones sociales tendría que ver más con un monto de ansiedad-timidez, que con retraimiento. En el factor 2 (autocontrol en las relaciones sociales) se observó una combinación de los ítems originales más otros tres (ítem 3, ítem 16 e ítem 59) de la dimensión consideración con los demás (Co), con un ítem (23) de la escala liderazgo de la versión española. Este resultado permite afirmar que los adolescentes evaluados consideraban que hablar con respeto hacia los adultos (ítem 3), saludar al entrar a un lugar (ítem 16), escuchar a los otros (ítem 59) y contribuir a que los trabajos sean más interesantes y variados (ítem 23) son habilidades vinculadas al autocontrol en las relaciones con los demás, más que a la consideración con los demás o al liderazgo. Consecuentemente, la escala Ac aparecía conformada por 18 ítems, es decir, con cuatro ítems más que en la versión española.

Los factores descritos mostraron niveles de fiabilidad aceptables, oscilando desde 0,520 a 0,721. La escala sinceridad mostró una consistencia interna de 0,608 , idéntico valor al señalado por Silva Moreno y Martorell Pallás (2001). A su vez, los coeficientes descritos en el presente estudio fueron similares a los análisis de fiabilidad realizados por Garaigordobil (2003) en una investigación sobre un programa de intervención socioemocional en población española de 10 a 12 años.

El segundo objetivo fue identificar las dimensiones de la socialización incluidas en el modelo jerárquico. El análisis factorial exploratorio mostró la presencia de dos factores que explicaban el $53,13 \%$ de la varianza de la BAS-3. Estos factores coincidían con las dimensiones de la socialización propuestas por Martorell et ál. (1995), que apuntan, por un lado, a la conducta prosocial y, por otro, a su polo negativo: la conducta antisocial, como también a la sociabilidad vs. baja sociabilidad. Tal como se plantea en la operacionalización del modelo jerárquico de la socialización, la conducta prosocial contenía las escalas Co y los ítems positivos de Ac, mientras que su polo negativo hacía lo propio con los ítems negativos de la escala Co. Por su parte, la bipolaridad sociabilidad vs. baja sociabilidad incluyó las escalas $L i$, en su polo positivo, y $\operatorname{Re}$ y At, en su polo negativo.

La varianza expuesta por esta solución factorial explica al menos un $50 \%$ de la variabilidad total de respuestas a la prueba autoinforme, condición que destacan Tornimbeni et ál. (2008). Los dos factores extraídos son coincidentes con las dimensiones del modelo jerárquico de la socialización y se verifican además por las correlaciones entre las escalas $\mathrm{Co}$ y $\mathrm{Ag}$, al igual que entre $\mathrm{Ac}$ y Ag (pertenecientes a la primera dimensión bipolar) y entre Re y At (de la segunda dimensión).

Las dimensiones de la socialización descritas destacan, según Kohn (1977), los problemas de conducta y personalidad, particularmente en el polo negativo de dichas dimensiones. Los problemas de conducta, tales como la agresividad y la dominancia, tienden a ser más estables y con consecuencias más significativas, puesto que pueden cristalizarse en la vida adulta en comportamientos delictivos y antisociales. El comportamiento antisocial refiere una amplia gama de manifestaciones comportamentales que incluyen robar, mentir, vagar, desafiar a la autoridad, entre otros, en los que se violan principios, normas y expectativas sociales (Angel de Grieff, Gaviria y Restrepo, 2003). Desde lo psicopatológico, este tipo de problemática se ubica como externalizante (Achenbach, 1995), caracterizada por agresión, impulsividad, desafío, rasgos antisociales y exceso de actividad.

La dimensión sociabilidad vs. baja sociabilidad refiere, según Kohn (1977), figuran problemas de 
personalidad, tales como la timidez y el retraimiento. Los puntajes promedio en esta dimensión pusieron en evidencia la tendencia del grupo adolescente participante a mostrar déficits para establecer relaciones socialmente satisfactorias con pares y adultos. Se trataría de un grupo de adolescentes que presenta un patrón de conductas caracterizado por un déficit o inadecuación de relaciones interpersonales y una gran tendencia a evitar los contactos sociales con otras personas en diversas situaciones sociales. Sin embargo, Portela (2009) reconoce que la incomodidad social moderada es común, especialmente frente a personas no conocidas o de un estatus social o autoridad superior. Para esta autora, una preocupación moderada acerca de la evaluación social puede permitir al individuo un desempeño óptimo en determinadas situaciones sociales.

Por otro lado, estos datos pueden referir una tendencia general del grupo social analizado, es decir, hacen alusión a características conservadoras de los grupos sociales del NOA (Argentina) que tienden a manifestar una actitud desconfiada hacia otras personas, por pertenecer a grupos desconocidos o diferentes. Al respecto, Garaigordobil (2000) encontró en un estudio con adolescentes de 12 a 14 años que aquellos que presentaban muchas imágenes negativas o prejuiciosas de otros adolescentes, según características como etnia o religión, presentaban asimismo muchas conductas de retraimiento social respecto a sus pares. Arce et ál. (2008) destacan que la base de los comportamientos socialmente positivos se relaciona con el desarrollo de habilidades que apuntan a considerar la perspectiva del otro, generando la posibilidad de construir redes sociales promotoras de integración.

Un análisis aparte merece la escala sinceridad de la BAS-3. Su inclusión, tal como plantean Silva Moreno y Martorell Pallás (2001), se vincula al cuestionario de personalidad EPQ de Eysenck y Eysenck (1989). Según Eysenck, esta escala debe interpretarse como una escala de conformidad con las normas sociales, más concretamente con la deseabilidad social. Según Paulhus (2002), la deseabilidad social tiene dos aspectos bien definidos: el autoengaño y el manejo de impresiones.
Este último se define como una tendencia a adoptar intencionalmente la imagen pública de uno mismo, con el propósito de obtener una visión favorable de los demás. Por el contrario, el autoengaño es la tendencia no intencionada de uno mismo a describirse de un modo favorable, lo que se manifiesta en autodescripciones sesgadas positivamente, pero en las que se cree honestamente (Salgado, 2005). Según Silva, Martínez y Ortet (1997), la escala S tendría una inclinación más hacia el manejo de la propia imagen. Las correlaciones positivas encontradas entre esta escala y la polaridad conducta antisocial y la escala Ag destacan un factor de resistencia y oposición a las normas sociales establecidas, hallándose una asociación negativa entre la dimensión conducta prosocial y S. Este dato llama la atención puesto que la consideración con los demás, el respeto y el autocontrol son constructos socialmente deseables. Según González (1992), la escala S debería entenderse junto a $\mathrm{Ag}$, puesto que apunta a la no-conformidad con las normas sociales.

Los datos psicométricos obtenidos de la BAS-3 destacan varias cuestiones. En primer lugar, que la fiabilidad de las escalas Co, Ac, Li y Re son moderadas, mientras que la At es aceptable; el reducido número de ítems, particularmente en $\mathrm{Li}$, podría estar influyendo en los índices de confiabilidad. En segundo lugar, la solución factorial de estas escalas permite comprobar la propuesta de Silva Moreno et ál. (1997) respecto al modelo jerárquico de la socialización. Este modelo plantea dos dimensiones bipolares: conducta prosocial vs. antisocial y sociabilidad vs. baja sociabilidad. Las dimensiones encontradas en el presente estudio muestran coherencia respecto a los factores primarios que las componen, puesto que se hallaron asociaciones estadísticas entre las escalas que constituyen la primera dimensión y asociaciones estadísticas negativas respecto a las escalas integrantes de la segunda dimensión. En tercer lugar, las asociaciones estadísticas encontradas entre estas dimensiones primarias y secundarias destacan la organización de este modelo jerárquico, con un repertorio de habilidades sociales en su base y un indicador propiamente dicho de la socialización en su extremo superior. 
Más allá de las limitaciones de los indicadores psicométricos encontrados, la versión de la BAS3 analizada en este estudio ha demostrado tener propiedades psicométricas acordes con la escala original, y muestra la utilidad para su uso en población adolescente local. Sin embargo, se sugieren ciertas modificaciones respecto a la versión original española: por un lado, la agrupación de los ítems en torno a los factores-escalas, según su saturación en el mencionado factor; por otro, la adecuación de aquellos ítems que aparecen como transparentes respecto al constructo que se investiga (por ejemplo, ítem 34: soy violento y golpeo a los demás). Estas modificaciones podrían conducir a disminuir los índices de deseabilidad social y a aumentar la fiabilidad del instrumento.

Futuras investigaciones sobre esta escala deberían evaluar la estabilidad en el tiempo de las puntuaciones a través de la confiabilidad testretest. Asimismo, sería recomendable evaluar las propiedades psicométricas del instrumento y ampliar los intervalos de edad de los adolescentes de Tucumán.

\section{Referencias}

Achenbach, T. (1992). New developments in multiaxial empirically based assessment of child and adolescent psychopathology. En J. Rosen \& P. McReynolds (Dirs.). Advances in Psychological Assessment, 8, 75-102. New York: Plenum.

Achenbach, T. (1995). Empirically based assessment and taxonomy: applications to clinical research. Psychological Assessment, 7, 261-274.

Achenbach, T. \& Edelbrock, C. (1978). The classification of child psychopathology: a review and analysis of empirical efforts. Psychological Bulletin, 85, 1275-1301.

Ángel de Grieff, E., Gaviria, P. y Retrepo, S. (2003). La conducta agresiva y su relación con la conducta antisocial. En A. Silva (Ed.).
Conducta antisocial: un enfoque psicológico (pp. 101-148). México: Pax.

Arce, S., Cordera, M., Perticarari, M., Villagarcía, M. et ál. (2008, noviembre). Los valores prosociales en el contexto de la violencia. Boletín de Psicología Interamericana, 89. Recuperado de http://boletin.sipsych.org/index.php?id_ page $=12 \&$ todo $=$ showpage\&idt $x t=78$.

Bandura, A. (1976). Social learning theory. New Jersey: Prentice Hall.

Caballo, V. (2005). Manual de evaluación y entrenamiento de las habilidades sociales (6. ${ }^{\mathrm{a}}$ ed.). Madrid: Siglo XXI.

Campbell, D. T. \& Fiske, D. W. (1959). Convergent and discriminant validation by the multitraitmultimethod matrix. Psychological Bulletin, 56(2), 81-105.

Carretero-Dios, H. \& Pérez, C. (2007). Standards for the development and review of instrumental studies: considerations about test selection in psychological research. International Journal of Clinical and Health Psychology, 7, 863-882.

Cohen, R. \& Swerdlik, M. (2001). Pruebas y evaluación psicológicas. Introducción a las pruebas y a la medición. México: McGraw-Hill.

Cordero, A. (Dir.) (1997). La evaluación psicológica en el año 2000. Madrid: TEA.

Curran, J. P. (1979). Pandora's Box reopened? The assessment of social skills. Journal of Behavioral Assessment, 1, 55-71.

Denzin, N. (1978). The research act: a theoretical introduction to sociological methode. New York: McGraw-Hill.

Eysenck, H. y Eysenck, S. (1989). EPQ Cuestionario de personalidad para niños (EPQ-J) y adultos (EPQ-A). Manual. Madrid: TEA. 
Figueroa Masip, A. (2006). Evaluación multimétodo y multiinformante de la sintomatología depresiva en niños y adolescentes (tesis doctoral). Universidad de Barcelona. Recuperado de http://www.tdx.cat/bitstream/handle/10 803/2534/01.AFM_INTRO.pdf;jsessionid=C2B 1AF0B0612EA597F428D69B51171D1.tdx2? sequence $=2$

Forns i Santacana, M. (1993). Evaluación psicológica infantil. Barcelona: Barcanova.

Garaigordobil Landazabal, M. (2000). Un estudio correlacional de las cogniciones prejuiciosas con diversas conductas sociales y con rasgos de personalidad. Anuario de Psicología Universidad de Barcelona, 3(31), 39-57.

Garaigordobil Landazabal, M. (2003). Intervención psicológica para desarrollar la personalidad infantil: juego, conducta prosocial y creatividad. Madrid: Pirámide.

Giraldo Alarcón, F. (2010). Propuesta de educación de las habilidades sociales de los estudiantes de IED Nueva Constitución que han tomado los talleres de Componente de Convivencia del Programa Comportamiento Social y Salud (tesis doctoral). Bogotá: Universidad Nacional de Colombia. Recuperado de http://es.scribd. com/doc/79970462/propuesta-de-evaluacion-de-las-habilidades-sociales-de-los-estudiantes-del-ied-nuevaconstitucion-que-hantomado-lostalleres-del-componente-conviv

Gismero, E. (2000). Escala de habilidades sociales. Madrid: TEA.

González, M. (1992). Conducta prosocial: evaluación e intervención. Madrid: Morata.

González, P. y Pelechado Barberá, V. (1996). Programa integrado de entrenamiento en habilidades interpersonales para ciclo medio. En V. Pelechano (Dir.). Habilidades interpersonales: teoría mínima y programas de intervención (vol. II, pp. 351-382). Valencia: Promolibro.
Hernández Plaza, S., Pozo Muñoz, C. \& Alonso Morillejo, E. (2004). The role of in-formal social support in needs assessment: proposal and application of a model to assess immigrants' needs in the south of Spain. Journal of Community and Applied Social Psychology, 14(4), 284-298.

Ipiña, M., Molina, L. y Reyna, C. (2011). Propiedades psicométricas de la Escala MESSY (versión autoinforme) en niños argentinos. Revista de Psicología, 29(2), 245-264.

Kirchner, T. (1997). Evaluación del desarrollo psicosocial. En G. Buela Casal y C. Sierra (Dir.). Manual de evaluación psicológica: fundamentos, técnicas y aplicaciones (pp. 773-778). Madrid: Siglo XXI.

Kohn, M. (1977). The Kohn Social Competence Scale and Kohn Symptom Checklist for the Preschool Child: a follow-up report. Journal of Abnormal Child Psychology, 3(5), 249-260.

Lacunza, A. (2007). Inteligencia y desnutrición. La evaluación de las habilidades cognitivas y sociales en niños de Tucumán en contextos de pobreza (tesis doctoral). Buenos Aires: Universidad de Palermo.

Lacunza, A. (2010, noviembre). Habilidades sociales y comportamiento prosocial en los niños. Propuestas de evaluación e intervención. Trabajo presentado en el $\mathrm{V}$ Encuentro Iberoamericano de Psicología Positiva. Buenos Aires.

Maganto Mateo, C. y Cruz Sáez, S. (2001). Multitécnicas y multimétodo en evaluación de la primera infancia. RIDEP, 2(12), 149-184

Martorell, M. (1997). Evaluación de la conducta social. En M. Casullo (Comp.). Evaluación psicológica en el campo socioeducativo (pp. 245301). Buenos Aires: Paidós.

Martorell, M., González, R., Aloy, M. y Ferris, M. (1995). Socialización y conducta prosocial. 
Revista Iberoamericana de Diagnóstico y Evaluación Psicológica, 1, 73-102.

Matson, J. L., Rotatori, A. F. \& Helsel, W. J. (1983). Development of a rating scale to measure social skills in children: the Matson Evaluation of Social Skills with Youngsters (MESSY). Behavior Research and Therapy, 27(4), 335-340.

Michelson, L., Sugai, D., Wood, R. y Kazdin, A. (1987). Las habilidades sociales en la infancia: evaluación y tratamiento. Barcelona: Martínez Roca.

Monjas Casares, M. (2002). Programa de Enseñanza de Habilidades de Interacción Social (Pehis) para niños y niñas en edad escolar. Madrid: CEPE.

Montero, I. y León, O. G. (2005). Sistema de clasificación del método en los informes de investigación en psicología. International Journal of Clinical and Health Psychology, 5, 115-127.

Murphy, G., Murphy, L. \& Newcomb, T. (1937). Experimental social psychology: an interpretation of research upon the socialization of the individual. New York: Harper \& Brothers.

Paulhus, D. (2002). Socially desiable responding: the evolution of a construct. En H. Braun \& D. Jackson (Eds.). Role of contructs in psychological and educational measurement (pp. 4969). Mahwah, New York: Lawrence Erlbaum Associates.

Pelechano Barberá, V. (1979). Psicología educativa comunitaria en E.G.B. Valencia: Alfaplús.

Pérez-Pareja, J. (1997). Autoinformes. En G. Buela Casal y C. Sierra (Dirs.). Manual de evaluación psicológica: fundamentos, técnicas y aplicaciones (pp. 297-314). Madrid: Siglo XXI.

Portela, A. (2009). Ansiedad social y trastorno evitativo de la personalidad: coexistencia y continuidad con otros trastornos psiquiátricos $y$ con rasgos y dimensiones de la personalidad.
ANXIA, Anuario de la Sociedad Argentina de Trastornos de Ansiedad, 81-92.

Reynolds, C. R. \& Komphaus, R. W. (1992). Behavior Assesmet System for Children (BASC). Manual. Circle Pines, MN: American Guidance.

Salgado, J. (2005). Personalidad y deseabilidad social en contextos organizacionales: implicaciones para la práctica de la psicología del trabajo y las organizaciones. Papeles del Psicólogo, 26, 115-128.

Sheinsohn, M., Uriel, F., Freiberg Hoffman, A. y Luchetti, Y. (2011). Adaptación y validación factorial de la versión para adolescentes de la escala de Autoeficacia Social EAS-U. En Actas del III Congreso Internacional de Investigación y Práctica Profesional en Psicología (pp. 137139). Buenos Aires.

Shelton, K., Frick, P. \& Wootton, J. (1996). The assessment of parenting practices in families of elementary scholl-aged children. Journal of Clinical Child Psychology, 25, 317-327

Silva Moreno, F. y López de Silva, M. (1979). Tipificación de las escalas AHS-1 y ETC-1 en niños canarios. Análisis y Modificación de la Conducta, 9, 223-244.

Silva Moreno, F. y Martorell Pallás, M. (1983). BAS 1-2, batería de socialización (para profesores y padres). Manual. Madrid: TEA.

Silva Moreno, F. y Martorell Pallás, M. (1991). La Batería de Socialización: nuevos datos sobre estructura y red nomológica. Psychological Assessment, 7(3), 349-367.

Silva Moreno, F. y Martorell Pállas, M. C. (2001). BAS-3, batería de socialización (autoevaluación). Madrid: TEA.

Silva Moreno, F., Martínez Arias, R. y Ortet, G. (1997). La evaluación de la orientación interpersonal: revisión de una línea de investigación. Revista 
de Psicología General y Aplicada, 50(1), 85-112.

Silva Moreno, F., Moro, M. y Ortet, G. (1994). Dimensiones de orientación interpersonal: un modelo y un instrumento. En M. Garaigordobil y C. Maganto (Dirs.). Socialización y conducta prosocial en la infancia y en la adolescencia (pp. 89-108). San Sebastián: Universidad del País Vasco.

Smulovitz, A. (2011). Validación de la Escala de Socialización BAS-3 en niños de 9 y 10 años de Concepción (Provincia de Tucumán) (tesis de licenciatura). Tucumán, Argentina:
Universidad del Norte Santo Tomás de Aquino.

Tornimbeni, S., Pérez, E. y Olaz, F. (2008). Introducción a la psicometría. Buenos Aires: Paidós.

Valero, L. (1997). La evaluación del comportamiento infantil: características y procedimientos. Apuntes de Psicología, 51, 51-79.

Williams, H. (1935). A factor analysis of Berne's social behavior in young children. Journal Experimental Education, 4, 142-146. 